\title{
Green's function for the one-dimensional Helmholtz equation: closed-form solution from its Fourier sine series
}

\author{
Antonio S. de Castro ${ }^{* 1}$ \\ ${ }^{1}$ Universidade Estadual Paulista "Júlio de Mesquita Filho", Departamento de Física, Guaratinguetá, SP, Brasil.
}

Received on February 21, 2021. Revised on March 22, 2021. Accepted on March 25, 2021.

It is presented a way to obtain the closed form for Green's function related to the nonhomogeneous onedimensional Helmholtz equation with homogeneous Dirichlet conditions on the boundary of the domain from its Fourier sine series representation. A closed form for the sum of the series $\sum_{k=1}^{\infty} \sin k x \sin k y /\left(k^{2}-\alpha^{2}\right)$ is found in the process.

Keywords: Green's function method, Nonhomogeneous Helmholtz equation, Homogeneous Dirichlet conditions.

Green's function method applied to differential equations is a topic crystallized in mathematical physics textbooks (see, e.g. 1-4) and has also appeared in some well-written didactic papers (see, e.g. [5] 9]). Green's function method is a tool suitable to solve nonhomogeneous differential equations, or homogeneous differential equations with nonhomogeneous initial or boundaryvalue conditions. Green's function satisfies a similar problem with the Dirac delta function taking place of the nonhomogeneous term. Green's function can be obtained from the linearly independent solutions of the corresponding homogeneous differential equation, and in some special circumstances its closed form can be obtained. Another way to obtain the Green function, specially useful when the homogeneous differential equation can not be solved by elementary methods, is by its expansion in a series of orthogonal functions. Green's function corresponding to the nonhomogeneous one-dimensional Helmholtz equation with homogeneous Dirichlet conditions prescribed on the boundary of the domain is an example of Green's function expressible in terms of elementary functions. Here, we review the Fourier series representation for this problem. Next we seek a closed form for such an infinite summation and show the equivalence with the closed-form Green's function obtained directly from the homogeneous equation.

The nonhomogeneous one-dimensional Helmholtz equation with homogeneous Dirichlet conditions on the boundary of the interval $(0, L)$ reads

$$
\begin{aligned}
\frac{d^{2} \psi(z)}{d z^{2}}+\kappa^{2} \psi(z) & =f(z) \\
\psi(0) & =\psi(L)=0
\end{aligned}
$$

This boundary-condition problem might describe the motion of a string with mass uniformly distributed along

\footnotetext{
${ }^{*}$ Correspondence email address: antonio.castro@unesp.br
}

its length, stretched along the $z$ axis from $z=0$ to $z=L$, and caused to vibrate by a harmonic force proportional to $f(z) \cos \kappa c t$. The small transverse displacement from its straight-line equilibrium position, $u(z, t)$, is governed by

$$
\begin{aligned}
\frac{\partial^{2} u(z, t)}{\partial z^{2}}-\frac{1}{c^{2}} \frac{\partial^{2} u(z, t)}{\partial t^{2}} & =f(z) \cos \kappa c t, \\
u(0, t) & =u(L, t)=0 .
\end{aligned}
$$

The constant $c$ (wave velocity) is equal to $\sqrt{T / \lambda}$, where $T$ and $\lambda$ are the tension and the linear uniform mass density of the string, respectively. The steady-state solution is written as $u(z, t)=\psi(z) \cos \kappa c t$, where $\psi(z)$ satisfies Eq. (1). The solution of Eq. (1) can be expressed as

$$
\psi(z)=\int_{0}^{L} G(z, \zeta) f(\zeta) d \zeta,
$$

where the Green function, $G(z, \zeta)$, satisfies

$$
\begin{aligned}
\frac{\partial^{2} G(z, \zeta)}{\partial z^{2}}+\kappa^{2} G(z, \zeta) & =\delta(z-\zeta), \\
G(0, \zeta) & =G(L, \zeta)=0 .
\end{aligned}
$$

Note that the solutions of the homogeneous onedimensional Helmholtz equation with homogeneous Dirichlet conditions are solutions of an eigenvalue problem of the self-adjoint operator $d^{2} / d z^{2}$ :

$$
\begin{aligned}
\frac{d^{2} \phi_{n}(z)}{d z^{2}} & =-\kappa_{n}^{2} \phi_{n}(z), \\
\phi_{n}(0) & =\phi_{n}(L)=0,
\end{aligned}
$$

with characteristic pair $\left(\kappa_{n}, \phi_{n}\right)$ equal to $(n \pi / L$, $\sin n \pi z / L)$. These eigenfunctions form a complete set of 
orthogonal functions over the interval $0 \leq z \leq L$ in such a way that is possible to represent $G(z, \zeta)$ by means of a series of these eigenfunctions:

$$
G(z, \zeta)=\sum_{n=1}^{\infty} g_{n}(\zeta) \sin \frac{n \pi z}{L} .
$$

Nevertheless, we have to determinate the unknowns Fourier coefficients $g_{n}(\zeta)$ to complete the specification of the Fourier sine series. Substitution of the series into Eq. (4) furnishes

$$
\sum_{n=1}^{\infty}\left(-\frac{n^{2} \pi^{2}}{L^{2}}+\kappa^{2}\right) g_{n}(\zeta) \sin \frac{n \pi z}{L}=\delta(z-\zeta) .
$$

Multiplying this series by $\sin \tilde{n} \pi z / L$, with $\widetilde{n}=$ $1,2,3, \ldots$, integrating term by term over $0 \leq z \leq L$, and taking advantage of the orthogonality relation

$$
\int_{0}^{L} d z \sin \frac{\tilde{n} \pi z}{L} \sin \frac{n \pi z}{L}=\frac{L}{2} \delta_{n \widetilde{n}},
$$

we find

$$
\sum_{n=1}^{\infty}\left(-\frac{n^{2} \pi^{2}}{L^{2}}+\kappa^{2}\right) g_{n}(\zeta) \delta_{n \tilde{n}}=\frac{2}{L} \sin \frac{\tilde{n} \pi \zeta}{L},
$$

in such a way that the Kronecker delta symbol $\delta_{n \tilde{n}}$ kills every term in the sum except the one for which $n=\widetilde{n}$. Then, we have

$$
\begin{aligned}
g_{n}(\zeta)= & -\frac{2 L}{\pi^{2}} \frac{1}{n^{2}-\kappa^{2} L^{2} / \pi^{2}} \sin \frac{n \pi \zeta}{L}, \\
& {[\kappa \neq n \pi / L] . }
\end{aligned}
$$

It follows that

$$
\begin{aligned}
& G(z, \zeta) \\
& =-\frac{2 L}{\pi^{2}} \sum_{n=1}^{\infty} \frac{1}{n^{2}-\kappa^{2} L^{2} / \pi^{2}} \sin \frac{n \pi \zeta}{L} \sin \frac{n \pi z}{L}, \\
& \quad[\kappa \neq n \pi / L] .
\end{aligned}
$$

To obtain the closed-form Green's function we use the trigonometric identity

$$
-\cos z_{1}+\cos z_{2}=2 \sin \frac{z_{1}+z_{2}}{2} \sin \frac{z_{1}-z_{2}}{2},
$$

and the closed-form formula (see 1.445(6) in Ref. [10])

$$
\begin{gathered}
\sum_{k=1}^{\infty} \frac{\cos k x}{k^{2}-\alpha^{2}} \\
=\frac{1}{2 \alpha^{2}}-\frac{\pi}{2} \frac{\cos \alpha[(2 m+1) \pi-x]}{\alpha \sin \alpha \pi} \\
{[(2 m) \pi \leq x \leq(2 m+2) \pi} \\
\alpha \text { is not an integer }]
\end{gathered}
$$

Then, we can write

$$
\begin{aligned}
\sum_{k=1}^{\infty} \frac{\sin k x \sin k y}{k^{2}-\alpha^{2}} & \\
= & \frac{\pi}{4 \alpha \sin \alpha \pi}\{-\cos \alpha[(2 m+1) \pi \mp(x-y)] \\
+ & \cos \alpha[(2 m+1) \pi-(x+y)]\} \\
& {[(2 m) \pi \leq x+y \leq(2 m+2) \pi} \\
& (2 m) \pi \leq \pm(x-y) \leq(2 m+2) \pi
\end{aligned}
$$

$\alpha$ is not an integer],

where, for the sake of simplicity, we have taken the arbitrary real parameter $m$ appearing in Eq. (13) the same as that one appearing in the second and third lines of Eq. (14). Note that the different signs in front of $(x-y)$ guarantee the invariance under a permutation of $x$ and $y$ on the right-hand side of Eq. (14), and the range of feasible values for $x$ and $y$ are determined by two different systems of inequalities depending on the sign in front of $(x-y)$. For convenience we isolate the variable $y$ in one of the systems of inequalities appearing in Eq. (14), viz.

$$
(2 m) \pi \leq x \pm y \leq(2 m+2) \pi,
$$

in such a way that

$$
\begin{aligned}
-x+(2 m) \pi & \leq y \leq-x+(2 m+2) \pi, \\
x-(2 m+2) \pi & \leq y \leq x-(2 m) \pi .
\end{aligned}
$$

The determination of the range of feasible values for $x$ and $y$ can be better understood by observing Fig. 1 . where the two lines of Eq. 16) are plotted on the same grid. The feasible values are represented by the shaded region where the plots overlap. It follows from such a plot that

$$
|y| \leq \pi, \quad|y|+(2 m) \pi \leq x \leq|y|+(2 m+2) \pi .
$$

Similarly, the system

$$
(2 m) \pi \leq y \pm x \leq(2 m+2) \pi,
$$

gives rise to

$$
|x| \leq \pi, \quad|x|+(2 m) \pi \leq y \leq|x|+(2 m+2) \pi .
$$

Therefore, using Eq. (12) again, the novel closed-form summation takes the form

$$
\begin{aligned}
\sum_{k=1}^{\infty} \frac{\sin k x \sin k y}{k^{2}-\alpha^{2}} & \\
= & \frac{\pi}{2} \frac{\sin \alpha[(2 m+1) \pi-y] \sin \alpha x}{\alpha \sin \alpha \pi}, \\
& \quad[|x| \leq \pi,(2 m) \pi+|x| \leq y \leq(2 m+2) \pi-|x|,
\end{aligned}
$$

$\alpha$ is not an integer,

similar formula is obtained by permuting $x$ and $y$ ]. 


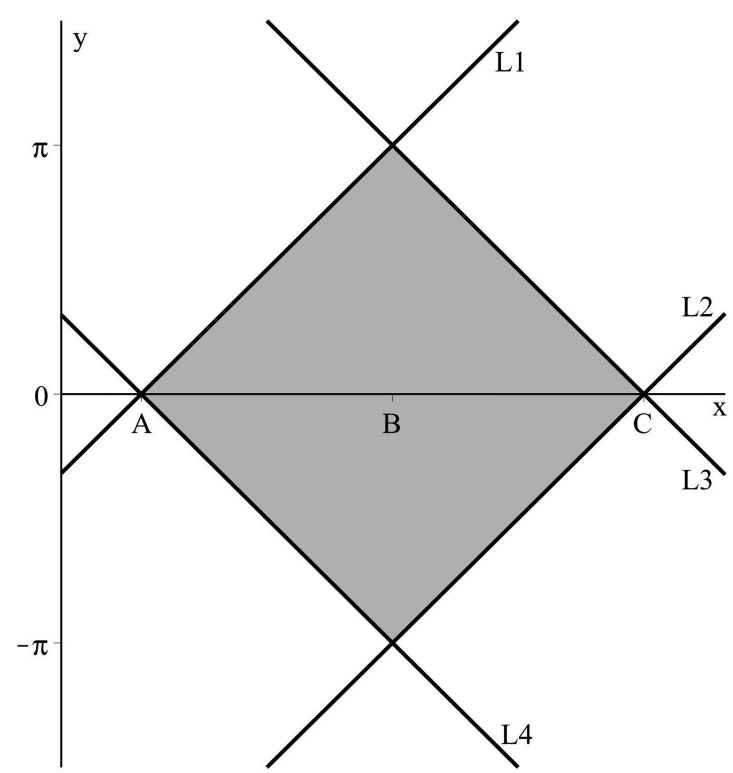

Figure 1: Solution to the system expressed by Eq. (16). The abscissas $A, B$ and $C$ stand for $(2 m) \pi,(2 m+1) \pi$ and $(2 m+2) \pi$, respectively. The lines $L 1, L 2, L 3$ and $L 4$ represent the functions $y=x-(2 m) \pi, y=x-(2 m+2) \pi$, $y=-x+(2 m+2) \pi$ and $y=-x+(2 m) \pi$, respectively. The shaded area represents the feasibile values of $x$ and $y$.

After the precedent digression into relevant details leading to Eq. 20, we are ready to obtain the closedform Green's function. In particular, with $m=0$, the substitutions $k \rightarrow n, \alpha \rightarrow \kappa L / \pi, x \rightarrow \pi z / L$ and $y \rightarrow \pi \zeta / L$ are sufficient to guarantee that $|z| \leq L$ with $|z| \leq \zeta \leq 2 L-|z|$, and $|\zeta| \leq L$ with $|\zeta| \leq z \leq 2 L-|\zeta|$ after permuting. Finally, remembering that the original problem is defined in the interval $0 \leq z \leq L$, with $0 \leq \zeta \leq L$, the closed form for the Green function expressed by Eq. 111 can be written as

$$
\begin{aligned}
G(z, \zeta)= & \left\{\begin{array}{l}
\frac{\sin \kappa(\zeta-L) \sin \kappa z}{\kappa \sin \kappa L}, 0 \leq z \leq \zeta, \\
\frac{\sin \kappa(z-L) \sin \kappa \zeta}{\kappa \sin \kappa L}, \zeta \leq z \leq L,
\end{array}\right. \\
& {[\kappa \neq n \pi / L] . }
\end{aligned}
$$

This result is in agreement with that one that follows in a well-known way from the knowledge of the exact solutions of the homogeneous one-dimensional Helmholtz equation. As a matter of fact, there is no need for a series expansion in this simple problem because the closed-form solution can be found in terms of elementary functions. Indeed, Eq. (21) is equivalent to Eq. (11). It is easy to verify that the Fourier sine series expansion of Eq. 21 leads to Eq. (11). Although one can not always expect to find a closed-form expression of a series expansion, this task is greatly facilitated in the present problem by the never seen before, to the best of author's knowledge, closed-form summation expressed by Eq. 20 .

\section{Acknowledgements}

The author would like to thank the anonymous referee for criticisms and suggestions. Grant 09126/2019-3, Conselho Nacional de Desenvolvimento Científico e Tecnológico (CNPq), Brazil.

\section{References}

[1] M.L. Boas, Mathematical Methods in the Physical Sciences (Wiley, New York, 1966), 2 ed.

[2] E. Butkov, Mathematical Physics (Addison-Wesley, Reading, 1968).

[3] G.B. Arfken and H.J. Weber, Mathematical Methods for Physicists (Harcourt/Academic Press, San Diego, 1996), 5 ed.

[4] K.F. Riley, M.P. Hobson and S.J. Bence, Mathematical Methods for Physics and Engineering (Cambridge University Press, Cambridge, 2006), 3 ed.

[5] J. Bellandi Filho, E.C. de Oliveira and H.G. Pavão, Rev. Bras. Ens. Phys. 6, 9 (1984).

[6] J. Bellandi Filho, R.J.M. Covolan, A.B. de Pádua and J.T.S. Paes, Rev. Bras. Ens. Phys. 10, 50 (1988).

[7] J. Bellandi Filho, R.J.M. Covolan, A.B. de Pádua and J.T.S. Paes, Rev. Bras. Ens. Phys. 11, 74 (1989).

[8] J. Bellandi Filho, R.J.M. Covolan, A.B. de Pádua and J.T.S. Paes, Rev. Bras. Ens. Phys. 12, 2 (1990).

[9] M.M. Odashima, B.G. Prado and E. Vernek, Rev. Bras. Ens. Phys. 39, e1303 (2017).

[10] I.S. Gradshteyn and I.M. Ryzhik, in: Table of Integrals, Series, and Products, edited by A. Jeffrey and D. Zwillinger (Academic Press, New York, 2007), 7 ed. 